\title{
Complete Genome Sequence of a Novel Ourmia-like Mycovirus Infecting the Phytopathogenic Fungus Botryosphaeria Dothidea
}

Liying Sun ( $\sim$ sunliying@nwsuaf.edu.cn )

Northwest Agriculture and Forestry University https://orcid.org/0000-0003-2538-6096

\section{Ziqian Lian}

Northwest Agriculture and Forestry University College of Plant Protection

\section{Subha Das}

Northwest Agriculture and Forestry University College of Plant Protection

Jingxian Luo

Northwest Agriculture and Forestry University College of Plant Protection

Ida Bagus Andika

Qingdao Agricultural University School of Agriculture and Plant Protection

\section{Research Article}

Keywords: Mycovirus, Ourmia-like virus, Botourmiaviridae, Botryosphaeria dothidea, Ascomycete, Fungi, Apple ring rot

Posted Date: May 19th, 2021

DOl: https://doi.org/10.21203/rs.3.rs-505029/v1

License: (9) (1) This work is licensed under a Creative Commons Attribution 4.0 International License. Read Full License 


\section{Abstract}

In this study, we describe the full-length genome sequence of a novel ourmia-like mycovirus, tentatively designated Botryosphaeria dothidea ourmia-like virus 1 (BdOLV1), isolated from the phytopathogenic fungus, Botryosphaeria dothidea strain $\mathrm{P} 8$, associated with apple ring rot in Shanxi province, China. The complete BdOLV1 genome is comprised of 2797 nucleotides, a positive-sense (+) single-stranded RNA (ssRNA) with a single open reading frame (ORF). The ORF putatively encodes a 642-amino acid polypeptide with conserved RNA-dependent RNA polymerase (RdRp) motifs, related to viruses of the family Botourmiaviridae. Phylogenetic analysis based on the RdRp amino acid sequences showed that BdOLV1 is grouped with oomycete-infecting unclassified viruses closely related to the genus Botoulivirus in Botourmiaviridae. This is the first report of a novel (+)ssRNA virus in B. dothidea related to the genus Botoulivirus in the family Botourmiaviridae.

\section{Introduction}

Botryosphaeria dothidea is a notorious canker pathogen that infects a wide range of trees worldwide [1]. This fungus is the principal causal agent of apple ring rot in China and is distributed across almost every apple planting region [2]. $B$. dothidea causes cankerous lesions on stems and brown rings on leaves and fruits, thereby ultimately hampering the apple yield and quality [3]. While fungicides are regularly applied for controlling this disease, the indiscriminate use of chemicals negatively impacts the environment and poses a threat to human health [3,4]. These concerns necessitate the development of alternative, environmentally friendly management strategies for preventing apple ring rot.

Mycoviruses or viruses that infect fungi are present throughout all major fungal taxa [5]. Mycoviruses are predicted to lack an extracellular phase, with their transmission occurring either vertically through conidia or spores, or horizontally via hyphal fusion followed by cytoplasmic mixing between compatible fungal strains [5]. Mycovirus genomes primarily consist of single- or double-stranded RNAs (ssRNA or dsRNA), although the recent discovery of circular ssDNA mycoviruses has increased their diversity $[5,6]$.

In general, mycoviruses cryptically infect their hosts, although some can diminish host virulence upon infection [7]. These viruses have the potential to be used as "virocontrol" agents for managing fungal diseases of plants. Mycovirus-infected debilitated strains can be introduced into a field to undergo hyphal fusion with their virulent counterparts, making them hypovirulent upon viral transmission. The first successful example of such mycovirus-mediated biocontrol was using Cryphonectria hypovirus 1 (CHV1) to control chestnut blight caused by Cryphonectria parasitica [7]. Several other mycoviruses have since been experimentally proven capable of introducing hypovirulence into their host fungi [5]. To explore mycoviral diversity in $B$. dothidea, extensive virus hunting in this pathogen has been conducted by numerous research groups. Such expeditions discovered several novel viruses in this fungus including members of the families Narnaviridae, Chrysoviridae, Fusariviridae, Totiviridae, Partitiviridae, and Botourmiaviridae [8-13]. 
Botourmiaviridae is a recently established linear positive-sense (+) ssRNA virus family comprising four recognized genera: Ourmiavirus, Botoulivirus, Scleroulivirus, and Magoulivirus [14]. The genus Ourmiavirus consists of plant-infecting viruses with encapsidated trisegmented genomes, where each segment separately encodes a movement protein, capsid protein, and RNA-dependent RNA polymerase (RdRp). In contrast, viruses belonging to the other three genera infect specifically fungi and oomycetes and are monosegmented with a single open reading frame (ORF) encoding an RdRp [14]

In this study, we report a novel (+) ssRNA ourmia-like mycovirus from $B$. dothidea strain $8 \mathrm{~A}$, which is associated with apple ring rot in China. Sequence comparison and phylogenetic analyses suggested that this virus is related to members of Botoulivirus in the family Botourmiaviridae and has been provisionally named Botryosphaeria dothidea Ourmia-like virus 1 (BdOLV1).

\section{Provenance of the virus in B. dothidea:}

B. dothidea strain 8A was originally isolated from an infected apple tree in Shanxi province, China. Upon establishing pure culture, the strain was maintained on potato dextrose agar (PDA) at $25^{\circ} \mathrm{C}$ under dark conditions. Strain identification was performed by internal transcribed spacer (ITS) sequencing as described by Xu and colleagues [15]. Total dsRNA (the replicative form of the virus) was extracted from a three-day-old mycelial culture grown on cellophane-overlaid PDA as described by Eusebio-Cope and Suzuki and visualized by $1 \%$ agarose gel electrophoresis in 1 X TAE buffer [16].

The partial CDNA sequence of BdOLV1 was initially obtained through RNA deep sequencing of ribosomal RNA depleted total RNA from strain 8A using Illumina platform. The full-length cDNA sequence of BdOLV1 was then obtained by amplifying its terminal regions adopting a 3' RNA ligase-mediated rapid amplification of cDNA ends (3' RLM-RACE) method. Briefly, a linker primer PC3-T7-loop (5'-pGGATCCCGGGAATTCGGTAATACGACTCACTATATTTTTATAGTGAGTCGTATTA-OH-3') was ligated to the 3 ' ends of heat-denatured $\left(95^{\circ} \mathrm{C}\right.$ for $\left.4 \mathrm{~min}\right)$ viral dsRNA at $4^{\circ} \mathrm{C}$ for $24 \mathrm{~h}$ using T4 RNA Ligase (Takara) following the manufacturer's instructions. The loop primer-linked purified dsRNA was then subjected to first-strand cDNA synthesis using SuperScript ${ }^{\mathrm{TM}}$ III Reverse Transcriptase (Invitrogen) with linker primer PC2 (5'-CCGAATTCCCGGGATCC-3'), complementary to the $5^{\prime}$ side of the PC3-T7-loop primer. To amplify $5^{\prime}$ and $3^{\prime}$ viral terminal regions, the resulting cDNA was then amplified using 2×Es Taq MasterMix (CWBIO) with complementary primer PC2 (5'-CCGAATTCCCGGGATCC-3') and gene-specific primers 406R (5'AAACCAGGGGCGAAAGCACGAC-3') and 2546F (CGAACTGCTGAGTCGGGGTGAT), respectively. The PCR products were subsequently cloned using pGEM®-T Easy Vector System I (Promega). For each RACE reaction, a minimum of three recombinant plasmids was sequenced in both directions using universal primers M13F and M13R.

The partial viral sequence and all terminal sequences were assembled and analyzed using DNAMAN version 9.0 (Lynnon Biosoft). The identity of BdOLV1 and its similarity to other viruses was determined via online BLAST analyses (https://blast.ncbi.nlm.nih.gov/Blast.cgi). The position of the ORF on the BdOLV1 genome and its corresponding putative polypeptide were determined using the ORF finder program (http://www.ncbi.nlm.nih.gov/gorf/orfig.cgi). Sequence alignments and phylogenetic analyses 
were performed using the MEGA version 10.1.7 software package [17]. The complete genome of BdOLV1 was submitted to GenBank under accession no. MZ073729.

\section{Sequence properties:}

The complete genome of BdOLV1 is 2797 nucleotides (nt) in length with a GC content of $54.06 \%$ (Fig. 1a). The 5'- and 3'-untranslated regions (UTRs) are 68 and 800 nt long, respectively (Fig. 1a). BdOLV1 contains a single ORF of 1929 nt, putatively encoding a 642-amino acid polypeptide with a deduced molecular mass of $76.62 \mathrm{kDa}$ (Fig. 1a).

A BLASTP analysis showed that this polypeptide is related to the RdRps of several ourmia-like viruses characterized from oomycetes and fungi. BdOLV1 RdRp was found to share $98.91 \%, 56.94 \%$, and $46.51 \%$ sequence identity with the corresponding regions of Botryosphaeria dothidea Ourmia-like virus (BdOLV, unpublished partial genome sequence), Plasmopara viticola lesion associated ourmia-like virus 54 , and Plasmopara viticola lesion associated ourmia-like virus 2, respectively. Despite a lack of conserved domains in CD-Search, multiple sequence alignment of the putative RdRp region from BdOLV1 with corresponding regions of other Botourmiaviridae members showed the presence of eight conserved RdRp motifs including a highly conserved GDD signature (on motif $\mathrm{VI}$ ) on the BdOLV1 polypeptide (Fig. 1b). Collectively, the findings suggest that BdOLV1 is a novel ourmia-like virus in the family Botourmiaviridae.

The generic identity of BdOLV1 was further determined using a Maximum Likelihood phylogenetic tree constructed from partially conserved RdRp sequences (Fig. 2). The tree topology showed that BdOLV1 grouped (100\% bootstrap support) with previously reported ourmia-like viruses from the oomycete Plasmopara viticola and ascomycete fungi B. dothidea and Phaeoacremonium minimum (Fig. 2). Interestingly, this group of viruses showed phylogenetic relatedness to another ourmia-like viruses group ( $99 \%$ bootstrap support) belonging to the genus Botoulivirus in the family Botourmiaviridae (Fig. 2).

Notably, pairwise sequence alignment of full-length RdRp amino acid sequences between BdOLV1 and Botrytis ourmia-like virus (Botrytis botoulivirus), an exemplar strain of the genus Botoulivirus, showed only $34.85 \%$ sequence identity, far below the current set species criteria $(\leq 90 \%)$ within this genus. Moreover, the complete RdRp sequences for members of different genera within Botourmiaviridae differ by $>70 \%$ [14]. At present, it is phylogenetically difficult to conclude whether BdOLV1 and its closely related viruses are novel species within the genus Botoulivirus or whether they constitute a new genus in the family Botourmiaviridae.

In this study, we characterized BdOLV1 from an apple-infecting ascomycete fungus $B$. dothidea showing no apparent disease symptoms. BdOLV1 differs from the previously characterized ourmia-like virus, Botryosphaeria dothidea botourmiavirus 1 (BdBOV-1), which was isolated from a hypovirulent pearinfecting $B$. dothidea strain [10]. While BdBOV-1 is phylogenetically related to the genus Magoulivirus, BdOLV1 is related to the genus Botoulivirus in the family Botourmiaviridae. Interestingly, BdOLV1 shares a close association with several ourmia-like viruses infecting oomycete $P$. viticola, suggesting a probable exchange of such ourmia-like viruses between fungi and oomycetes. Notably, both $B$. dothidea and $P$. 
viticola are tree pathogens, suggesting that both organisms may have acquired such viruses from a common source, and viruses thereafter evolved with their respective hosts.

\section{Declarations}

\section{Acknowledgments:}

We greatly thank Dr. Guangyu Sun for kindly providing research materials. This work was supported in part by National Natural Science Foundation of China (30970163) to LY. Sun and (31970159), to IB. Andika.

\section{Compliance with ethical standards}

\section{Conflict of interest:}

All authors declare that they have no conflicts of interest.

\section{Ethical approval:}

This article does not contain any studies with human participants or animals performed by any of the authors.

\section{References}

1. Marsberg A, Kemler M, Jami F, et al (2017) Botryosphaeria dothidea: a latent pathogen of global importance to woody plant health. Mol Plant Pathol 18:477-488.

2. Tang W, Ding Z, Zhou ZQ, et al (2011) Phylogenetic and pathogenic analyses show that the causal agent of apple ring rot in China is Botryosphaeria dothidea. Plant Dis 96:486-496.

3. Brown-Rytlewski DE, McManus PS (2000) Virulence of Botryosphaeria dothidea and Botryosphaeria obtusa on apple and management of stem cankers with fungicides. Plant Dis 84:1031-1037.

4. Nicolopoulou-Stamati P, Maipas S, Kotampasi C, et al (2016) Chemical Pesticides and Human Health: The urgent need for a new concept in agriculture. Front public Heal 4:148.

5. Ghabrial SA, Castón JR, Jiang D, et al (2015) 50-plus years of fungal viruses. Virology 479480:356-368.

6. Li P, Wang S, Zhang L, et al (2020) A tripartite ssDNA mycovirus from a plant pathogenic fungus is infectious as cloned DNA and purified virions. Sci Adv 6:eaay 9634.

7. Nuss DL (1992) Biological control of chestnut blight: an example of virus-mediated attenuation of fungal pathogenesis. Microbiol Rev 56:561- 576

8. Wang H, Liu H, Lu X, et al (2021) A novel mitovirus isolated from the phytopathogenic fungus Botryosphaeria dothidea. Arch Virol 166:1507-1511. 
9. Liu W, Hai D, Mu F, et al (2020) Molecular characterization of a novel fusarivirus infecting the plantpathogenic fungus Botryosphaeria dothidea. Arch Virol 165:1033-1037.

10. Yang M, Zhou X, Zhai L, et al (2020) Molecular characterization of a novel mycovirus infecting the phytopathogenic fungus Botryosphaeria dothidea. Arch Virol 165:1667-1670.

11. Ding Z, Zhou T, Guo L-Y (2017) Characterization of a novel strain of Botryosphaeria dothidea chrysovirus 1 from the apple white rot pathogen Botryosphaeria dothidea. Arch Virol 162:20972102.

12. Zhai L, Hong N, Zhang M, Wang G (2015) Complete dsRNA sequence of a novel victorivirus isolated from the pear stem wart fungus Botryosphaeria dothidea. Arch Virol 160:613-616.

13. Wang L, Jiang J, Wang Y, et al (2014) Hypovirulence of the phytopathogenic fungus Botryosphaeria dothidea: association with a coinfecting chrysovirus and a partitivirus. J Virol 88:7517-7527.

14. Turina M, Hillman BI, Izadpanah K, et al (2017) ICTV Virus Taxonomy Profile: Ourmiavirus. J Gen Virol 98:129-130.

15. Xu C, Wang C, Sun X, et al (2013) Multiple group I introns in the small-subunit rDNA of Botryosphaeria dothidea: implication for intraspecific genetic diversity. PLoS One 8:e67808

16. Eusebio-Cope A, Suzuki N (2015) Mycoreovirus genome rearrangements associated with RNA silencing deficiency. Nucleic Acids Res 43:3802-3813.

17. Kumar S, Stecher G, Li M, et al (2018) MEGA X: Molecular evolutionary genetics analysis across computing platforms. Mol Biol Evol 35:1547-1549.

\section{Figures}


a

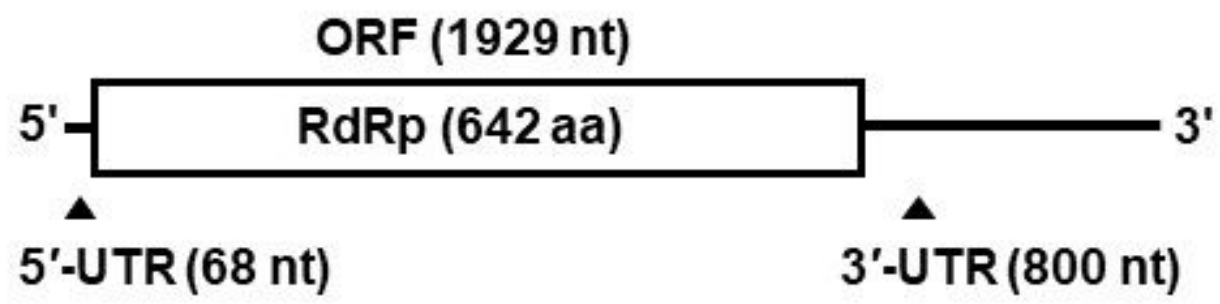

b

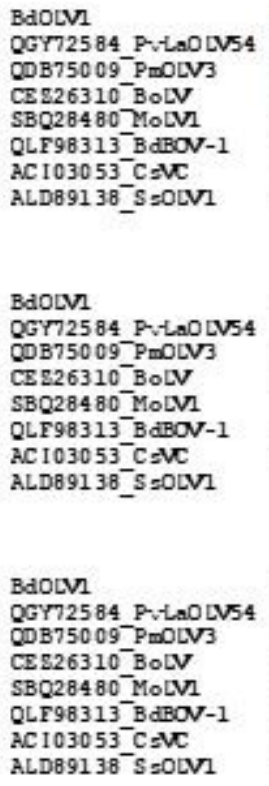

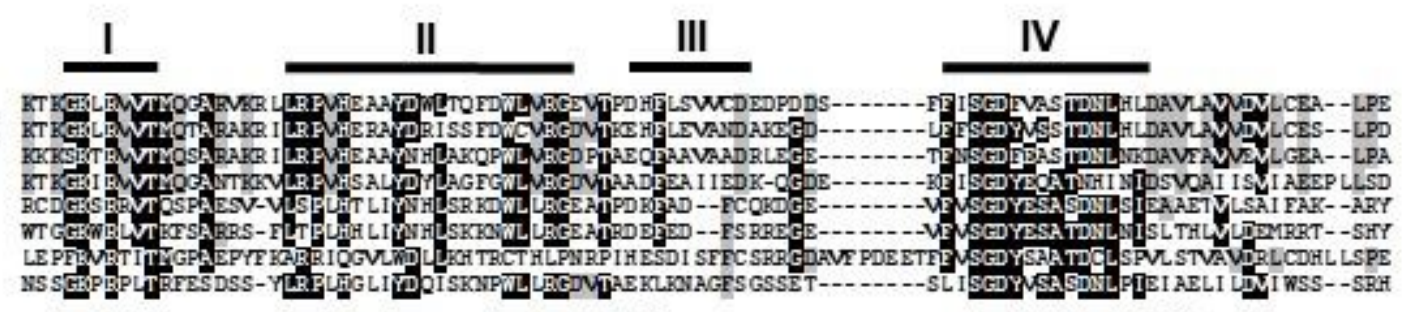
V $\quad$ VI

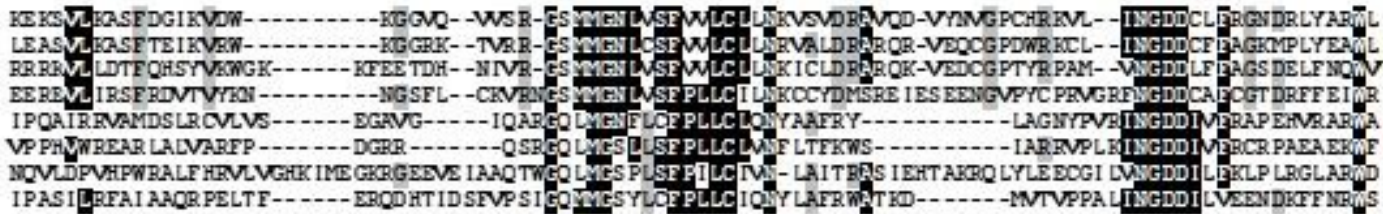

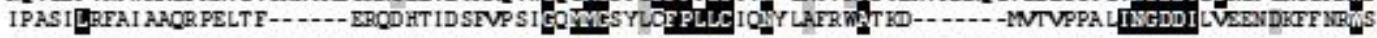

\section{VIII}

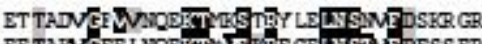

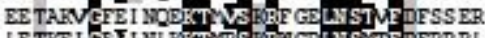

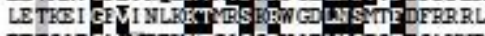

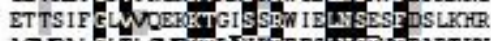

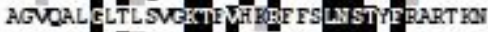

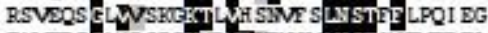

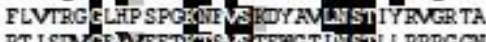

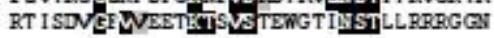

\section{Figure 1}

(a) Genetic organization of Botryosphaeria dothidea ourmia-like virus 1 (BdOLV1). The position of the open reading frame (ORF) is indicated by an open box. The length of the ORF and its corresponding polypeptide (RdRp) are indicated on top of and within the box (within parentheses), respectively. The 5'and 3 '-untranslated regions (UTR) are indicated by black arrows, with their corresponding lengths indicated within parentheses. (b) Amino acid sequence alignment of conserved RdRp motifs of BdOLV1 and selected members of different established or proposed genera within the family Botourmiaviridae. ClustalW was used to align amino acid sequences. Highly and less conserved amino acid positions are indicated by black shading and white lettering, and gray shading and black lettering, respectively. Motif positions (I to VIII) are indicated by straight black lines on top of each motif. Virus names with their corresponding genus identities are: Plasmopara viticola lesion associated ourmia-like virus 54 (PvLaOLV54), unassigned; Phaeoacremonium minimum ourmia-like virus 3 (PmOLV3), unassigned; Botrytis ourmia-like virus (BoLV), Botoulivirus; Magnaporthe oryzae ourmia-like virus 1 (MoLV1), Magoulivirus; Botryosphaeria dothidea botourmiavirus 1 (BdBOV-1), Magoulivirus; Cassava virus C (CsVC), Ourmiavirus; and Sclerotinia sclerotiorum ourmia-like virus 1 (SsOLV1), Scleroulivirus. 
Genus

Family

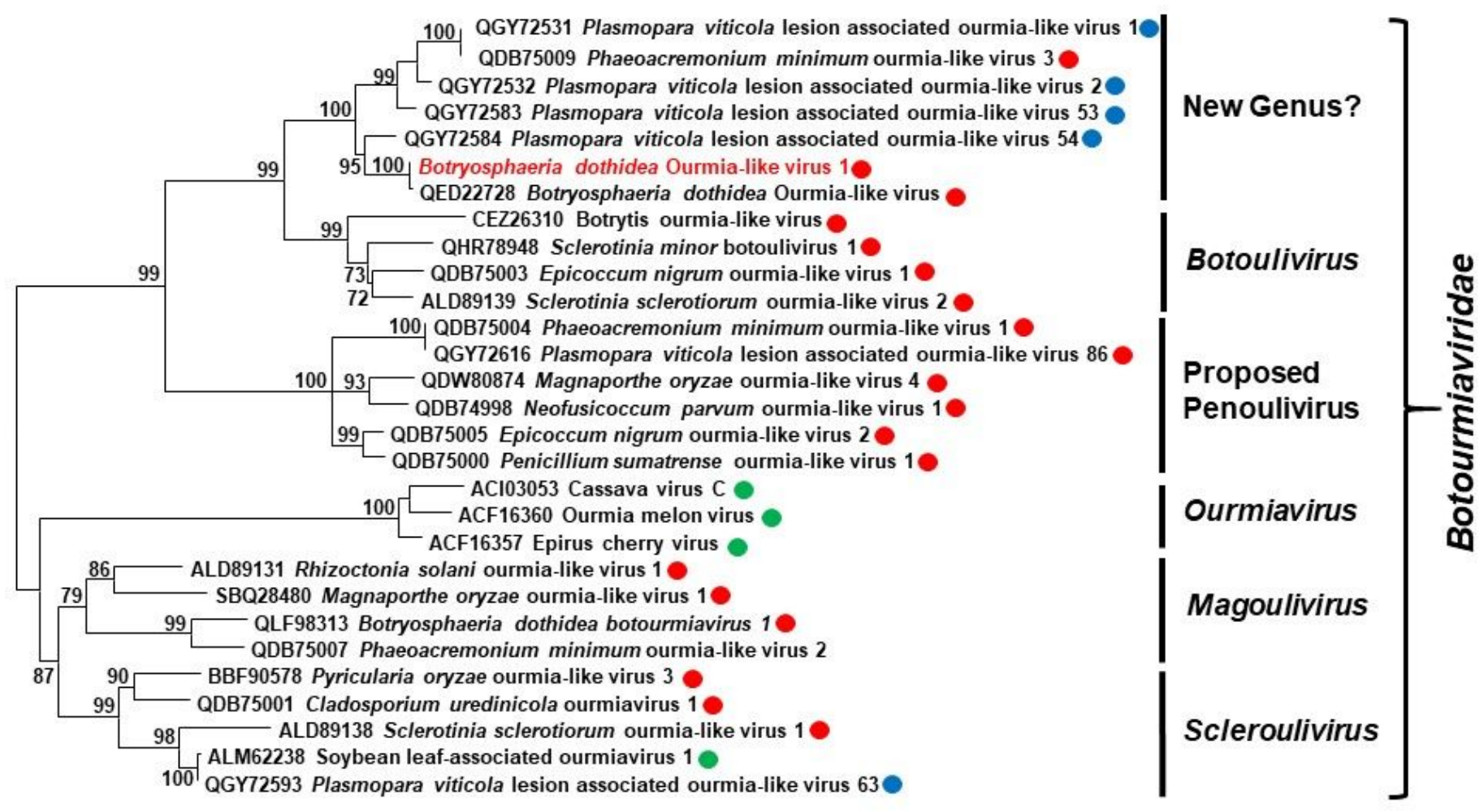

$\stackrel{\vdash}{\longleftarrow .50}$

Figure 2

A Maximum Likelihood phylogenetic tree (using LG model) constructed using the amino acid sequences of conserved RdRp regions of BdOLV1 (highlighted in red) and selected members of the family Botourmiaviridae (corresponding GenBank accession numbers are adjacent to virus names). A discrete gamma distribution was used to model evolutionary rate differences among the sites. The numbers next to each branch reflect the percentages of congruent clusters in 500 bootstrap replications. Bootstrap values $\leq 70 \%$ are not shown. Scale bar indicates a genetic distance of 0.5 amino acid substitution/site. Red, blue, and green filled circles adjacent to virus names indicate viral hosts (fungus, oomycete, and plant).

\section{Supplementary Files}

This is a list of supplementary files associated with this preprint. Click to download.

- BdOLV1completecDNA2797.doc 